\title{
INtraoperative photoDYnamic Therapy for GliOblastomas (INDYGO): study protocol for a phase I clinical trial
}

(Short title: Intraoperative Photodynamic Therapy for GBM)

\author{
Clément Dupont ${ }^{1}, \mathrm{PhD}$, Maximilien. Vermandel ${ }^{*}, 1,2, \mathrm{PhD}$, Henri-Arthur Leroy ${ }^{1,2}, \mathrm{MD}$, \\ Mathilde Quidet ${ }^{1,2}$, MD, Fabienne Lecomte ${ }^{1}$, MSc, Nadira Delhem ${ }^{3}$, PhD, Serge Mordon ${ }^{1}$, \\ $\mathrm{PhD}$, Nicolas Reyns ${ }^{1,2}, \mathrm{MD}, \mathrm{PhD}$,
}

1-Univ. Lille, Inserm, CHU Lille, U1189 - ONCO-THAI - Image Assisted Laser Therapy for Oncology, F-59000 Lille, France

2-Univ. Lille, CHU Lille, Department of Neurosurgery, F-59000 Lille, France

3- Univ. Lille, CNRS, UMR 8161, Institut de biologie de Lille, Institut Pasteur de Lille, 59000 Lille, France

${ }^{*}$ Corresponding author: maximilien.vermandel@,chru-lille.fr

C. Dupont and M. Vermandel contributed equally to the manuscript and are both co-first authors.

Conflict of interest: None.

Disclosure of funding: The authors of this publication have research support from INSERM, University of Lille, University Hospital of Lille, American Society for Laser In Medicine and Surgery, Ligue contre le Cancer and Agence Nationale pour la Recherche.

Acknowledgements: The authors would like to thank Estelle GALLET and Laurent JEAUNEAU from B. Braun (Boulogne-Billancourt, France) for providing the extraperitoneal distension balloons (Aesculap, Tuttlingen, Germany) used to design our lighting device free of charge.

Abstract (250 words, should summarize all the key elements of the protocol including the rationale, objectives, methods, populations, time frame, and expected outcomes):

\section{Background:}

Glioblastoma (GBM) is characterized by marked proliferation, major infiltration and poor prognosis. Despite current treatments, including surgery, radiation oncology and chemotherapy, the overall median survival is 15 months and the progression-free survival is 7 to 8 months. Because of systematic relapse of the tumor, the improvement of local control 
remains an issue. In this context, photodynamic therapy (PDT) may offer a new treatment modality for GBM.

\section{Objective:}

The main objective of the INDYGO clinical trial is to assess the feasibility of intraoperative PDT early after surgical resection of GBM without unacceptable and unexpected toxicities.

\section{Methods:}

This treatment will be carried out in addition to the current standard of care of glioblastoma: maximum resection surgery followed by concomitant radio-chemotherapy and adjuvant chemotherapy. PDT treatment will be delivered during surgery early, after the fluorescence guided resection. Immunological responses and biomarkers will also be investigated during the follow-up. A total of 10 patients will be recruited during this study.

\section{Expected Outcomes:}

Clinical follow-up after the standard of care with PDT is expected to be similar (no significant difference) to the standard of care alone.

\section{Discussion:}

This INDYGO trial assesses the feasibility of intraoperative 5-ALA PDT, a novel seamless approach to treat GBM. The technology is easily embeddable within the reference treatment at a low-incremental cost. The safety of this new treatment modality is a preliminary requirement before a multicenter randomized clinical trial can be further conducted to assess local control improvement by treating infiltrating and non-resected GBM cells.

\section{Trial registration:}

NCT number: NCT03048240

EudraCT number: 2016-002706-39

Keywords: 5-ALA; photodynamic therapy; fluorescence-guided resection; glioblastoma; neurosurgery; medical device; laser therapy

\section{General Information:}

Protocol title, protocol identifying number (if any), and date.

INtraoperative photoDYnamic Therapy of GliOblastoma (INDYGO), NCT03048240, registered on 9 February 2017

Name and address of the sponsor/funding agency.

University Hospital, Lille, 2 Avenue Oscar Lambret, 59000 Lille

Name and title of the investigator(s) responsible for conducting the research, and the address and telephone number(s) of the research site(s), including responsibilities of each. 
Pr. Nicolas Reyns, MD, PhD, Principal Investigator, Department of Neurosurgery, CHU Lille, INSERM OncoThAI U1189, +33320446721 nicolas.reyns@ @ chru-lille.fr

Dr. Maximilien Vermandel, Co-Principal investigator, PhD, Department of Medical Physics, CHU Lille, INSERM OncoThAI U1189, +33320446721 maximilien.vermandel@ chru-lille.fr

\section{Name(s) and address(es) of the clinical laboratory(ies) and other medical department(s) or institutions involved in the research.}

Institut National de la Santé Et de la Recherche Médicale, 1, Avenue Oscar Lambret, 59000 Lille, France

\section{Rationale and Background Information:}

Glioblastoma (GBM) is a brain tumor with a particularly poor prognosis. The median overall survival is approximately 15 months with the current standard of care (SOC) ${ }^{1-3}$. Although GBM is a rare neoplastic disease with low prevalence (3-5/100,000 persons), it remains the most frequent primary malignant brain tumor in adults ${ }^{4,5}$. Currently, no existing therapeutic agent is able to stop GBM progression. Complete resection is rarely feasible since isolated tumor cells usually infiltrate the surrounding brain. Adjuvant therapies to improve local control are thus highly expected.

Currently, the European Society of Medical Oncology (ESMO) guidelines provide recommended treatment options according to the grade of the glioma ${ }^{6}$. Given the invasive nature of GBM, local relapse is unavoidable. Radiotherapy (RT) applied to the tumor bed with concomitant and adjuvant chemotherapy with temozolomide (TMZ) complete the surgery and prolong the progression-free survival (PFS) and the overall survival (OS). Although these adjuvant treatments statistically improve outcomes, the benefits remain modest. An increase of less than 3 months in the median OS with RT and TMZ versus RT alone has been observed ${ }^{3}$. Therefore, the management of newly diagnosed patients should include a maximal resection when achievable; the extent of resection (EOR) is of prognostic value. To optimize the EOR, surgery commonly goes along with an intraoperative assistance to diagnosis residual tumor tissues.

Fluorescence-guided resection (FGR) with 5-aminolevulinic acid (5-ALA) as a prodrug uses the fluorescence phenomenon to distinguish healthy tissue from tumor tissue in order to support the surgical procedure. Due to a defective regulatory process in tumor cells, exogenous 5-ALA induces the accumulation of a photosensitive molecule, protoporphyrin IX (PpIX), in tumor cells only. When the surgery cavity is illuminated under blue light (375-440 $\mathrm{nm})$, the tumor tissues shine red $(635 \mathrm{~nm}$ ) (see figure 1). An increase in the EOR and an improvement in PFS has been observed when 5-ALA and is used for FGR ${ }^{7}$. However, it has been shown that extended resections performed using FGR carry the risk of temporary impairment of neurological function ${ }^{8}$. Furthermore, relapse occurs in the cavity margin in $85 \%$ of cases ${ }^{9}$. The improvement of local control by EOR and additional therapy delivered intraoperatively is a key point in increasing progression-free survival and overall survival ${ }^{10}$. In this context, intraoperative photodynamic therapy (PDT) appears relevant in treating areas bordering the resection cavity while maintaining a maximal but safe resection. 

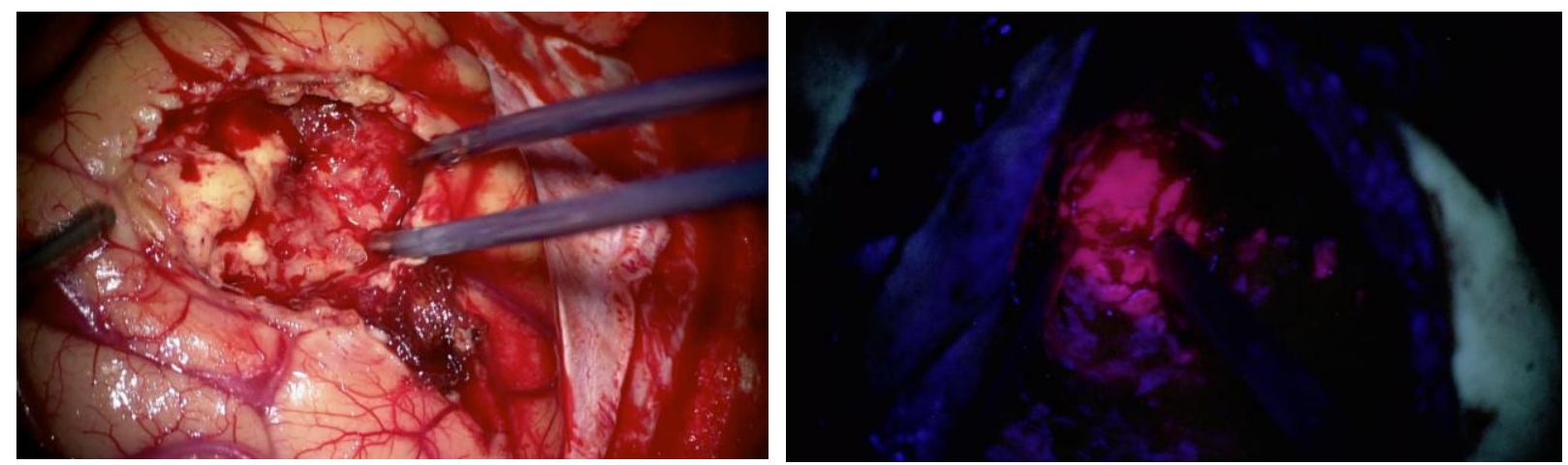

Figure 1: Fluorescence-guided resection under visible light (a) and blue light (b). Residual tumor cells shine red due to the fluorescence property of PpIX.

PDT is based on the photochemical reaction activated by light between a photosensitive molecule and oxygen. This reaction creates reactive oxygen species, including singlet oxygen, which leads to cell death. The selective accumulation of PpIX in tumor cells only makes this treatment local ${ }^{11-13}$. In addition to 5-ALA being used for intraoperative diagnosis, previous human experiences using 5-ALA as a prodrug for PDT have been reported and highlight the positive role of PDT for the treatment of GBM ${ }^{12,14,15}$. Additionally, an immune response seems to be triggered during PDT treatment, which would allow a delayed therapeutic action on $\mathrm{GBM}^{16,17}$.

Illumination of the resection cavity remains challenging since the device has to suit the shape of the cavity. In this context, a deformable balloon is expected to meet this requirement. Several devices have been designed for this purpose in the past, such as an inflatable balloon with different light sources ${ }^{18-22}$, an optical fiber coupled to a reflector, or a laser light source coupled to the surgical microscope, enabling a step by step illumination ${ }^{23}$. Moseley et al. described the only clinical study using 5-ALA as a precursor for the treatment of GBM with PDT ${ }^{20,21}$. For this study, two photosensitizers, 5-ALA and Photofrin ${ }^{\circledR}$ (Pinnacle Biologics, Chicago, IL), were considered, and repetitive PDT was evaluated. 5-ALA was used for the FGR procedure, and PpIX was activated during the first illumination. Following the procedure, further illuminations (post-operative) were applied to activate Photofrin ${ }^{\circledR}$, which has a longer half-life than 5-ALA. Thus, none of these previous studies used 5-ALA as a primary photosensitizer precursor to provoke a PDT effect on areas bordering the resection cavity, as proposed in the present study.

To be more acceptable for both the surgeons and the patients, intraoperative PDT has to be fully embedded within the usual surgical workflow. Consequently, the concept of this new therapeutic approach was to deliver 5-ALA PDT intraoperatively early after FGR.

This study adopts a repurposing strategy, using 5-ALA in a therapeutic manner for glioma, and hence develops a model for future therapeutic strategies for treating high-grade gliomas combined with the current SOC in addition to intraoperative 5-ALA PDT. Intraoperative PDT takes advantage of the presence of the photosensitizer, which is already approved and administered to the patient for the FGR procedure ${ }^{24}$. Indeed, even if no fluorescence is observed after FGR, remaining tumor cells around and deeper in the cavity are still present and take up PpIX at a concentration, limiting their visualization through the 
microscope. These remaining cells that are not microscopically visible are the main target of the PDT treatment that is delivered by means of a new, recently designed lighting device ${ }^{25}$.

The proposed treatment strategy can fit into the current SOC and can be applied at the initiation of care (see figure 2$)^{6}$. Thus, intraoperative 5-ALA PDT is more ethically acceptable compared with the use of interstitial PDT on the resectable tumor.

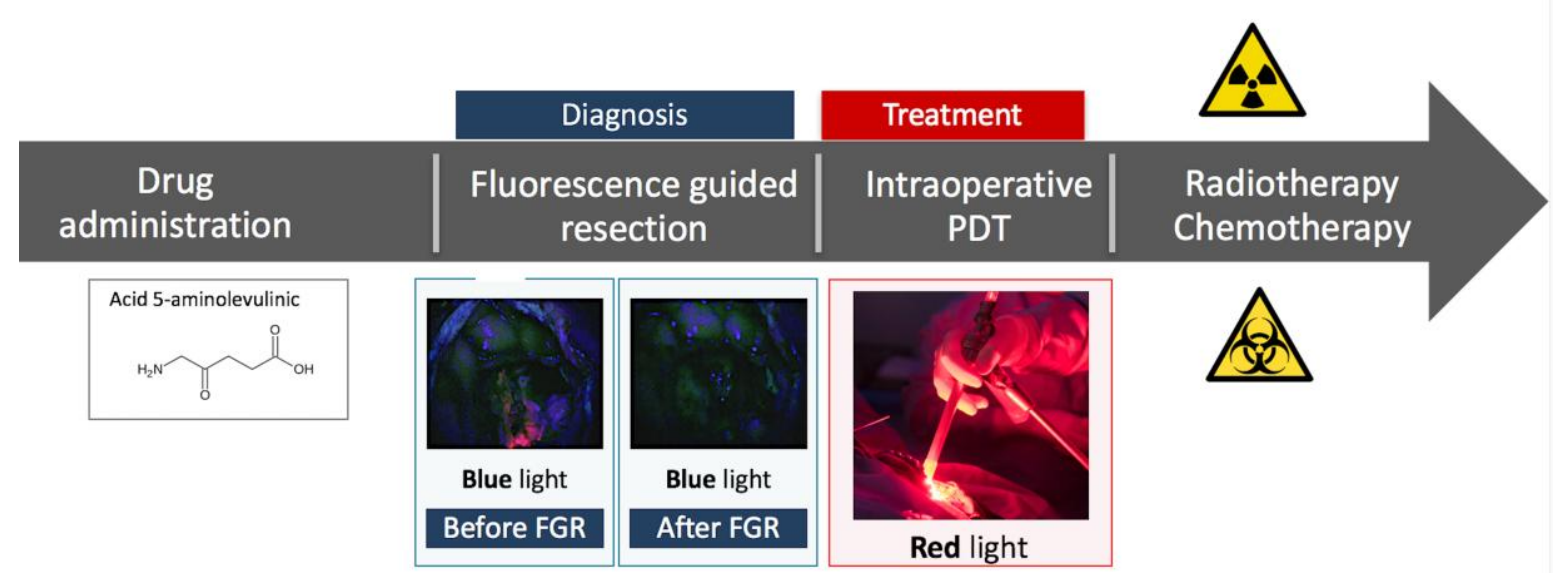

Figure 2: Workflow of the intraoperative PDT procedure

\section{Study Goals and Objectives}

The current INDYGO study aims to assess the feasibility and safety of the intraoperative 5ALA-mediated PDT procedure in patients with a newly diagnosed GBM that is accessible for complete surgical removal with contrast enhancement. The primary endpoint is to assess the feasibility of intraoperative PDT early after the surgical resection of GBM without unacceptable and unexpected toxicities.

The secondary endpoints are the evaluation of the following:

- progression-free survival,

- overall survival,

- treatment response evaluated by MRI every three months until tumor relapse,

- toxicity evaluated every three months until tumor relapse and follow-up of serious unexpected events potentially linked to patient death,

- quality of life measured every three months until tumor relapse.

To validate the study, at least seven out of the ten patients included in the study must be free of unacceptable and unexpected toxicities.

An immunological study has been added to the workflow to explore biological markers of the PDT- induced immune response.

\section{Study Design}

This study is an interventional, single group, pilot study and single center (university hospital of Lille, France). Enrollment of 10 patients is expected. All patients with newly-diagnosed probable GBM based on clinical and radiological criteria and in whom surgery is achievable could be recruited. The main exclusion criteria are contraindications to 5-ALA or MRI procedures. 


\section{Methodology}

A simplified workflow of the study is presented in figure 3. Once a patient is included in the INDYGO trial, MRI (T1, T1-weighted, T2, FLAIR, diffusion/perfusion sequences) and blood samples for immunology measurements are acquired. Next, 5-ALA is orally administered 4 hours before the surgery $(20 \mathrm{mg} / \mathrm{kg})$, and a standard surgical resection is performed, followed by the FGR procedure. Once there is no remaining fluorescence or potentially infiltrating functional areas, an intraoperative MRI is performed to assess the quality of the resection. After the MRI examination, the PDT procedure starts. During the setup of the device, a quality control evaluation of the laser is performed. The lighting device is placed inside the surgical cavity and is inflated with diffusing liquid until it reaches the cavity boundaries. The treatment time is deduced from the quantity of diffusing liquid injected in the device. The treatment sequence is composed of five fractions of illumination separated by two-minute breaks to re-oxygenate brain tissues. Soon after the PDT treatment is completed, a second intraoperative MRI and blood samples are acquired to assess potential early and acute effects of $\mathrm{PDT}^{26}$.

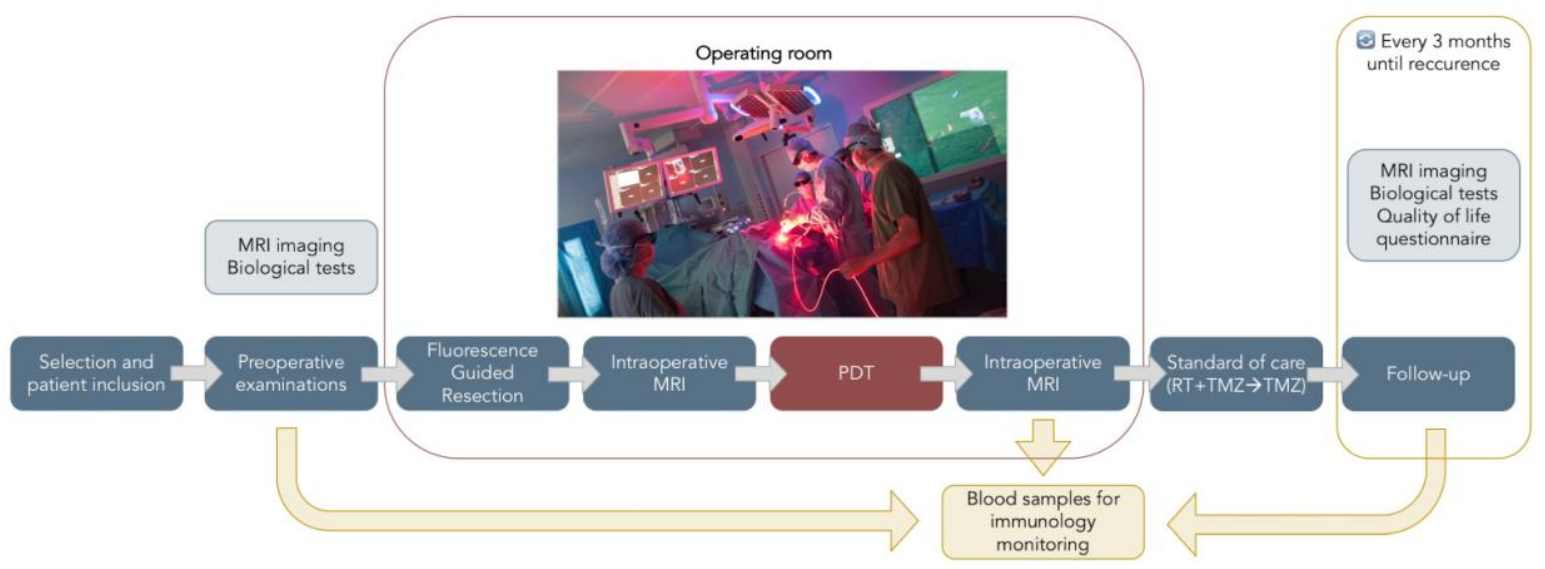

Figure3: Flowchart of the INDYGO trial

After surgery, patients undergo an SOC, including ${ }^{6}$ RT and concomitant TMZ, followed by adjuvant TMZ. MRI and blood samples are then acquired every three months alongside patient follow-up until recurrence.

Alongside this study, biological samples are collected and enable an exploratory study. This collection will be used to design future immune monitoring for each patient. Several parameters will be explored, such as measurements of cytokines, growth factors, immune cell profiles and peripheral blood mono-nucleated cells.

\section{Discussion}

The main difficulty of this clinical trial relies on the use of a new medical device simultaneously with the evaluation of a drug repurposed for this condition. This issue was raised due to a comprehensive assessment of this new medical device ${ }^{25}$. Thus, once the safety of the medical laser system combined with the medical device was evaluated in a preclinical stage, a risk management procedure was delivered to an institutional review board for ethical 
consideration and to the French National Medicine Agency for legal consideration. Additionally, a comprehensive analysis of the previous use of 5-ALA for the purpose of PDT associated with an efficacy and safety preclinical trial in a rodent model ${ }^{26}$ validated the repurposing of the drug for therapy. Managing both the medical device and the drug issues remain specific for PDT, and both have to be considered before being approved for human use.

\section{Trial Status}

The INDYGO clinical trial is still in the recruitment phase.

\section{Safety Considerations}

For each patient recruited, all the adverse events are recorded from the beginning of PDT treatment to relapse, up to 24 months. This collection of adverse events is reviewed by an independent safety monitoring board twice: in the middle and at the end of the study. The independent safety monitoring board is composed of three neurosurgeons from different university hospitals in France. Additionally, as the primary endpoint of this trial is the safety of PDT, this safety board composes also the data monitoring committee.

\section{Follow-up}

Several monitoring visits are planned after the PDT treatment, as recommended with the SOC6. RT and TMZ sequences are still administered to all patients. The INDYGO trial utilizes blood sample collections and MRI imaging every 3 months until recurrence.

A quality of life questionnaire has been established to investigate the impact of PDT treatment on the patient's quality of life (EORTC QLQ-C301). This collection is filled in every 3 months until relapse, by the signature of a consent form, and is assessed up to 24 months. This questionnaire includes a global health status and a quality of life scale.

\section{Data Management and Statistical Analysis}

Since this is a pilot feasibility study, in agreement with the statistical board, the number of subjects is not calculated on the basis of the statistical hypothesis. A total of 10 patients will be recruited over a planned period of one year. The feasibility objective is to have a minimum of seven patients included who have been fully treated with PDT, without unacceptable and unexpected toxicity.

Furthermore, this number of 10 patients seems compatible with the average number of patients recruited annually in the investigative center for the surgical treatment of GBM.

\footnotetext{
${ }^{1}$ http://groups.eortc.be/qol/eortc-qlq-c30
} 
All the data are described individually using case reports. If possible, a Kaplan-Meier curve will be established for the PFS. The analysis of the data will be carried out with the methodological support platform of Lille University Hospital.

\section{Quality Assurance}

All the medical observations are kept in the patient's file; the data regarding the study are reported on case report forms (CRF) according to good clinical practice. Any deviation from the protocol must be noted, and the reason for deviation must be documented. The data collection will be exhaustive and checked regularly by a clinical research nurse, according to the protocol procedures.

The monitoring of the trial is carried out according to the monitoring plan. A planning meeting with the principal investigator has been held before the start of the trial.

During the trial, several checkpoints will be defined, including the presence of signed informed consent forms obtained by the neurosurgeon, respect of the inclusion and exclusion criteria, the reporting of any adverse event and the monitoring of all steps of the patient's follow-up.

At the end of the trial and once the final analysis is completed and validated, all the files and data will be sealed and archived, according to specific procedures, in a secure location.

\section{Expected Outcomes of the Study}

The main expected result of this INDYGO study is the feasibility and safety assessment of intraoperative PDT. This is a crucial requirement to consolidate data prior to a multicenter randomized clinical trial. As secondary objectives, the study aims to demonstrate the potential effects of PDT in terms of progression-free survival and to provide new data on the effectiveness of PDT by radiological biomarkers.

From the patient side, the translational impact is highly relevant. Local control is highly expected, and PDT brings new solutions to its optimization. Adding PDT to the GBM SOC is expected to significantly increase progression-free survival.

\section{Duration of the Project}

Enrollments are scheduled for a period of 12 months, and follow-up is scheduled for 24 months.

\section{Project Management}

Pr. Reyns is the principal investigator of the study and will submit the final report. Dr. Vermandel is the scientific manager of the project. Pr. Mordon is the scientific expert. They will coordinate the publication plan to communicate trial results.

\section{Ethics}

The authors state that they have obtained appropriate institutional review board approval and have followed the principles outlined in the Declaration of Helsinki for all human or animal experimental investigations. In addition, for investigations involving human subjects, informed consent are collected from the participants involved. Access to the data are in agreement with the French National Agency regulating Data Protection (Commission Nationale Informatique et Libertés, CNIL). 
1. Iacob G, Dinca EB. Current data and strategy in glioblastoma multiforme. J Med Life. 2009;2(4):386-393.

2. Ostrom QT, Bauchet L, Davis FG, et al. The epidemiology of glioma in adults: a "state of the science" review. Neuro Oncol. 2014;16(7):896-913.

3. Stupp R, Mason WP, van den Bent MJ, et al. Radiotherapy plus concomitant and adjuvant temozolomide for glioblastoma. $N$ Engl J Med. 2005;352(10):987-996.

4. Grill J. Glioblastoma. 2007; http://www.orpha.net/consor4.01/www/cgibin/Disease_Search.php?lng=EN\&data_id=3752\&Disease_Disease_Search diseaseGr oup $=$ glioblastome\&Disease Disease Search diseaseType=Pat\&Maladie(s)/groupes $\%$

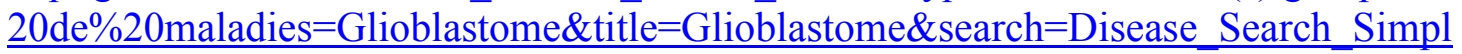
e.

5. Fisher JL, Schwartzbaum JA, Wrensch M, Wiemels JL. Epidemiology of brain tumors. Neurol Clin. 2007;25(4):867-890, vii.

6. Stupp R, Brada M, van den Bent MJ, Tonn JC, Pentheroudakis G, Group EGW. Highgrade glioma: ESMO Clinical Practice Guidelines for diagnosis, treatment and followup. Ann Oncol. 2014;25 Suppl 3:iii93-101.

7. Stummer W, Pichlmeier U, Meinel T, Wiestler OD, Zanella F, Reulen HJ. Fluorescence-guided surgery with 5-aminolevulinic acid for resection of malignant glioma: a randomised controlled multicentre phase III trial. The lancet oncology. 2006;7(5):392-401.

8. Stummer W, Tonn JC, Mehdorn HM, et al. Counterbalancing risks and gains from extended resections in malignant glioma surgery: a supplemental analysis from the randomized 5-aminolevulinic acid glioma resection study. Clinical article. $J$ Neurosurg. 2011;114(3):613-623.

9. Petrecca K, Guiot MC, Panet-Raymond V, Souhami L. Failure pattern following complete resection plus radiotherapy and temozolomide is at the resection margin in patients with glioblastoma. J Neurooncol. 2013;111(1):19-23.

10. Pavlov V, Page P, Abi-Lahoud G, et al. Combining intraoperative carmustine wafers and Stupp regimen in multimodal first-line treatment of primary glioblastomas. $\mathrm{Br} J$ Neurosurg. 2015;29(4):524-531.

11. Valdes PA, Kim A, Brantsch $M$, et al. delta-aminolevulinic acid-induced protoporphyrin IX concentration correlates with histopathologic markers of malignancy in human gliomas: the need for quantitative fluorescence-guided resection to identify regions of increasing malignancy. Neuro Oncology. 2011;13(8):846-856.

12. Johansson A, Faber F, Kniebuhler G, et al. Protoporphyrin IX fluorescence and photobleaching during interstitial photodynamic therapy of malignant gliomas for early treatment prognosis. Lasers in Surgery and Medicine. 2013;45(4):225-234.

13. Johansson A, Palte G, Schnell O, Tonn JC, Herms J, Stepp H. 5-Aminolevulinic acidinduced protoporphyrin IX levels in tissue of human malignant brain tumors. Photochem Photobiol. 2010;86(6):1373-1378.

14. Stummer W, Beck T, Beyer W, et al. Long-sustaining response in a patient with nonresectable, distant recurrence of glioblastoma multiforme treated by interstitial photodynamic therapy using 5-ALA: case report. Journal of Neurooncology. 2008;87(1):103-109.

15. Beck TJ, Kreth FW, Beyer W, et al. Interstitial photodynamic therapy of nonresectable malignant glioma recurrences using 5-aminolevulinic acid induced protoporphyrin IX. Lasers in Surgery and Medicine. 2007;39(5):386-393. 
16. Maeding N, Verwanger T, Krammer B. Boosting Tumor-Specific Immunity Using PDT. Cancers. 2016;8(10):91.

17. Wachowska M, Muchowicz A, Demkow U. Immunological aspects of antitumor photodynamic therapy outcome. Central-European Journal of Immunology. 2015;40(4):481-485.

18. Wilson BC, Muller PJ, Yanch JC. Instrumentation and light dosimetry for intraoperative photodynamic therapy (PDT) of malignant brain tumours. Physics in Medecine and Biology. 1986;31(2):125-133.

19. Dwyer PJ, White WM, Fabian RL, Anderson RR. Optical integrating balloon device for photodynamic therapy. Lasers in Surgery and Medicine. 2000;26(1):58-66.

20. Moseley H, McLean C, Hockaday S, Eljamel S. In vitro light distributions from intracranial PDT balloons. Photodiagnosis Photodyn Ther. 2007;4(3):213-220.

21. Eljamel MS, Goodman C, Moseley H. ALA and Photofrin fluorescence-guided resection and repetitive PDT in glioblastoma multiforme: a single centre Phase III randomised controlled trial. Lasers in medical science. 2008;23(4):361-367.

22. Schmidt MH, Bajic DM, Reichert KW, 2nd, Martin TS, Meyer GA, Whelan HT. Light-emitting diodes as a light source for intraoperative photodynamic therapy. Neurosurgery. 1996;38(3):552-556; discussion 556-557.

23. Akimoto J, Haraoka J, Aizawa K. Preliminary clinical report on safety and efficacy of photodynamic therapy using talaporfin sodium for malignant gliomas. Photodiagnosis Photodyn Ther. 2012;9(2):91-99.

24. Leroy HA, Vermandel M, Lejeune JP, Mordon S, Reyns N. Fluorescence guided resection and glioblastoma in 2015: A review. Lasers in surgery and medicine. 2015;47(5):441-451.

25. Dupont C, Mordon S, Deleporte P, Reyns N, Vermandel M. A novel device for intraoperative photodynamic therapy dedicated to glioblastoma treatment. Future Oncol. 2017.

26. Leroy HA, Vermandel M, Leroux B, et al. MRI assessment of treatment delivery for interstitial photodynamic therapy of high-grade glioma in a preclinical model. Lasers in surgery and medicine. 2017. 